\title{
Scale Interactions in Magnetohydrodynamic
}

\section{Turbulence}

\author{
Pablo D. Mininni \\ Departamento de Física, Facultad de Ciencias Exactas y Naturales, Universidad \\ de Buenos Aires and CONICET, Ciudad Universitaria, 1428 Buenos Aires, \\ Argentina, \\ and \\ NCAR, P.O. Box 3000, Boulder, Colorado 80307-3000, USA.
}
Key Words magnetohydrodynamics, modeling, simulation, isotropy, universal- ity

\begin{abstract}
This article reviews recent studies of scale interactions in magnetohydrodynamic turbulence. The present day increase of computing power, which allows for the exploration of different configurations of turbulence in conducting flows, and the development of shell-toshell transfer functions, has led to detailed studies of interactions between the velocity and the magnetic field and between scales. In particular, processes such as induction and dynamo action, the damping of velocity fluctuations by the Lorentz force, or the development of anisotropies, can be characterized at different scales. In this context we consider three different configurations often studied in the literature: mechanically forced turbulence, freely decaying turbulence, and turbulence in the presence of a uniform magnetic field. Each configuration is of interest for different geophysical and astrophysical applications. Local and non-local transfers are discussed
\end{abstract}


for each case. While the transfer between scales of solely kinetic or solely magnetic energy is local, transfers between kinetic and magnetic fields are observed to be local or non-local depending on the configuration. Scale interactions in the cascade of magnetic helicity are also reviewed. Based on the results, the validity of several usual assumptions in hydrodynamic turbulence, such as isotropy of the small scales or universality, is discussed.

\section{CONTENTS}

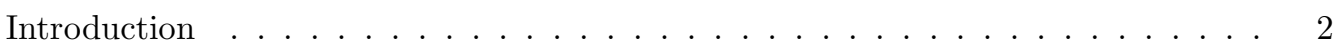

Indirect evidence of non-locality . . . . . . . . . . . . . . . . . 9

The shell-to-shell transfer .......................... . 11

Direct studies of multi-scale interactions . . . . . . . . . . . . . . . 14

Forced isotropic and homogeneous turbulence . . . . . . . . . . . . . 15

Freely decaying turbulence ................................ 18

Anisotropic turbulence ................................... 19

Magnetic helicity and the inverse cascade . . . . . . . . . . . . . . . . 21

Non-local interactions and universality of MHD turbulence . . . . . . . . . . 22

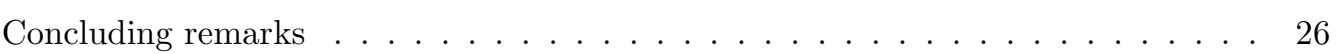

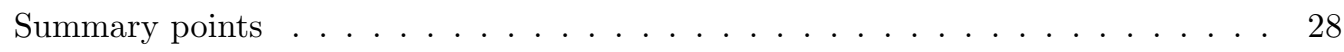

Acknowledgments . . . . . . . . . . . . . . . . . . . . . . . . . 29

\section{Introduction}

Turbulence is a multiscale phenomenon ubiquitous in geophysical and astrophysical flows. In many of these flows, the coupling of a conducting fluid with electromagnetic fields requires consideration of the magnetohydrodynamic (MHD) 
equations (see, e.g., Moffatt 1978). The equations describe the dynamics of nonrelativistic conducting fluids as, e.g., in the Earth's core or in industrial applications, and under some approximations they can also describe the large-scale behavior of magnetospheric, space, and astrophysical plasmas. In these latter cases, care must be taken to consider only the scales where a one-fluid approximation holds, as scales small enough may require consideration of kinetic plasma effects such as ambipolar diffusion in weakly ionized plasmas as the interstellar medium, or the Hall current for highly ionized media such as small scales in the solar wind. However, in those cases the MHD equations still give a good description of large scales, and the approximation gives a useful approach to get lowest-order physical insight into the fate of the flows.

In the simplest case, that of an incompressible flow with constant mass density, the equations give the evolution of the bulk fluid velocity $\mathbf{u}$ and of the magnetic field b:

$$
\begin{gathered}
\partial_{t} \mathbf{u}+\mathbf{u} \cdot \nabla \mathbf{u}=-\nabla p+\mathbf{b} \cdot \nabla \mathbf{b}+\nu \nabla^{2} \mathbf{u}, \\
\partial_{t} \mathbf{b}+\mathbf{u} \cdot \nabla \mathbf{b}=\mathbf{b} \cdot \nabla \mathbf{u}+\eta \nabla^{2} \mathbf{b},
\end{gathered}
$$

where the magnetic field is written in Alfvenic units, the density is set to unity, and $p$ is the (fluid plus magnetic) pressure. The kinematic viscosity $\nu$ and magnetic diffusivity $\eta$ control respectively the viscous and Ohmic dissipation. These equations are constrained by the incompressibility condition and by the solenoidal character of the magnetic field,

$$
\nabla \cdot \mathbf{u}=0, \quad \nabla \cdot \mathbf{b}=0
$$

Two different Reynolds numbers can be defined in MHD flows. The mechanical 
Reynolds number

$$
R e=\frac{U L}{\nu},
$$

which is the ratio of convective to viscous forces (where $U$ is the rms velocity and $L$ a characteristic lengthscale of the flow), and the magnetic Reynolds number

$$
R m=\frac{U L}{\eta}
$$

that can be interpreted as the ratio of induction to Ohmic dissipation. In many flows these Reynolds numbers are very large, and the flows are in a turbulent regime.

While in the hydrodynamic case the phenomenological theory of Kolmogorov (K41) gives to a good approximation (albeit intermittency corrections) the power law of the energy spectrum, no clearly established phenomenological counterpart exists in MHD. This has many implications as the energy dissipation rate (required to predict, e.g., heating rates in solar and space physics) depends on the slope of the energy spectrum. Also, subgrid models, required to do numerical modeling in astrophysics and geophysics given the large scale separation involved in such flows, are less developed in MHD as a result of the lack of detailed knowledge of its energy spectrum.

In the Kolmogorov description of hydrodynamic turbulence, the interactions of similar size eddies play the basic role of cascading the energy to smaller scales on a scale-dependent time scale $\tau_{\ell} \sim \ell / u_{\ell}$, where $\ell$ is the examined length scale and $u_{\ell}$ the characteristic velocity at that scale. This time scale, which is proportional to the eddy turnover time at the scale $\ell$, is the only time scale available on dimensional grounds in the inertial range, provided enough scale separation exists between forcing and dissipation. In this context, interactions between scales are local (in spectral space) as dominant interactions are between eddies of similar 
sizes. One then expects the statistical properties of sufficiently small scales to be independent of the way turbulence is generated, and to have therefore universal character. Recent experiments showed deviations from this behavior even for simple hydrodynamic flows (e.g., slower than expected recovery of isotropy, or presence of long-time correlations in the small scales; see Carlier et al. 2001, Poulain et al. 2006, Shen \& Warhaft 2000, Wiltse \& Glezer 1993, 1998). Numerical simulations also gave evidence of the presence of non-local interactions with the large scale flow playing a role in the cascade of energy (Alexakis et al. 2005a, Domaradzki 1988, Domaradzki \& Rogallo 1990, Zhou 1993). In numerical simulations with Reynolds numbers as high as $R_{\lambda} \approx 800$, it was observed that $20 \%$ of the energy flux in the small scales was due to interactions with the large scale flow (Mininni et al. 2006). However, more recent simulations with Reynolds numbers up to $R_{\lambda} \approx 1300$ using spatial resolutions of $2048^{3}$ grid points showed that as the Reynolds number is increased, the percentage of the non-local flux decreases as a power law of the Reynolds number, suggesting that the flux in hydrodynamic turbulence may be predominantly local for very large Reynolds number (Mininni et al. 2008). Recent theoretical results put this in firmer grounds (Aluie \& Evink 2009a, Eyink \& Aluie 2009), showing that the energy flux in hydrodynamic turbulence is local in the limit of infinite Reynolds number and obtaining bounds on the scaling of the non-local contribution to the flux with Reynolds number which are in agreement with the numerical results.

The case for MHD turbulence is less clear and has given rise to more controversies. Several attempts have been done to extend the phenomenological arguments of Kolmogorov to conducting flows (see, e.g., Boldvrev 2006, Goldreich \& Sridhar 1995, Iroshnikov 1963, Kraichnan 1965, Matthaeus \& Zhou 1989). However, the MHD 
equivalent of the $4 / 5$ law in hydrodynamic turbulence (the Politano-Pouquet relations, see Politano \& Pouquet 1998a,,$\underline{b})$ couple the velocity and the magnetic field in a way that can be compatible with several power law behaviors; in three dimensions they read

$$
\left\langle\delta z_{\|}^{\mp}(\mathbf{l})\left|\delta \mathbf{z}^{ \pm}(\mathbf{l})\right|^{2}\right\rangle=-\frac{4}{3} \epsilon^{ \pm} l
$$

where $\epsilon^{ \pm}$are the dissipation rates of the Elsässer variables $\mathbf{z}^{ \pm}=\mathbf{u} \pm \mathbf{b}$, and the subindex $\|$ denotes the increment of the field along the displacement vector $\mathbf{l}$.

Moreover, even in the simplest incompressible case, at least two time scales can be identified in the inertial range of MHD turbulence. Besides the eddy turnover time, incompressible MHD flows are also characterized by the period of Alfvén waves $\tau \sim\left(B_{0} L\right)^{-1}$, where $B_{0}$ is the amplitude of the large scale magnetic field in Alfvenic units. In a first attempt to derive a phenomenological theory, Iroshnikov (1963) and Kraichnan (1965) (IK) assumed that the large scale magnetic field acts as a uniform field for the small scale fluctuations, which then behave as Alfvén waves. In that case, small scales can interact not only through the eddies but also through Alfvén packages, which reduce the energy flux to small scales by increasing its transfer time. This introduces in practice a non-local interaction as the waves propagate along the large scale field (see Gomez et al. 1999 for a discussion). From dimensional analysis, Iroshnikov and Kraichnan then derived an isotropic energy spectrum proportional to $k^{-3 / 2}$. Later, extensions where considered to take into account the anisotropy induced at small scales by the large scale magnetic field (Boldyrev 2006, Galtier et al. 2000, 2005, Goldreich \& Sridhar 1995). Some of these extensions, after accounting for the anisotropy, rely on some form of a balance between the two fields that leaves only the turnover time as the relevant time scale, and can be therefore considered 
local or non-local depending on the authors.

At the core of the early disquisitions is the fact that in MHD the roles of a large scale flow and of a large scale magnetic field are different. While a (uniform) large scale flow can be removed by a Galilean transformation, a large scale magnetic field cannot. As a remarkable coincidence, lack of Galilean invariance is at the basis of the $\sim k^{-3 / 2}$ spectrum for hydrodynamic turbulence within the framework of the Direct Interaction Approximation (DIA) by Kraichnan (1959), a flaw later corrected by the development of the Test Field Model (TFM) and the Lagrangian History DIA (LHDIA). However, in MHD magnetic fields are not Galilean invariant and for this reason the associated Alfvén waves have to be taken into account in phenomenological theories, and are also considered when studying non-local effects in the Eddy-Damped Quasi-Normal Markovian (EDQNM) closure (Pouquet et al. 1976) or in weak turbulence theory (Nazarenko et al. 2001). However, although phenomenological descriptions assume that a large scale field has the effect of reducing the energy cascade rate, the transfer of energy (and the cascade) in many cases still takes place between eddies of similar size, presumably allowing for recovery of universal statistical properties at small scales.

In recent years, this universal behavior has been questioned by different authors. Because energy can be injected in MHD by a mechanical forcing or by an electromotive forcing, MHD turbulence is characterized by a larger number of regimes than hydrodynamic turbulence even in its simplest configurations. Magnetic fields in planets and stars are believed to be generated by dynamo action, where turbulent motions sustain magnetic fields against Ohmic dissipation (Brandenburg \& Subramanian 2005, Krause \& Raedler 1980, Moffatt 1978, Pouquet et al. 1976). This regime is often studied in numerical simulations (and 
recently in experiments, see Monchaux et al. 2007) by mechanically stirring the flow. Depending on the amount of mechanical helicity in the flow (the alignment between the velocity and the vorticity), or in the presence of large-scale shear, the magnetic field generated may have large or small-scale correlation (compared with the integral scale of the flow), giving a steady state that may be dominated by mechanical or magnetic energy. On the other hand, plasmas in the solar corona and in the solar wind are dominated by magnetic energy, and are often studied numerically by stirring the flow with electromotive forces or using simulations of freely decaying turbulence. Finally, the amount of cross-correlation between the velocity and the magnetic field depends on the flow (e.g., on the heliocentric distance in the solar wind) and can also be varied in the simulations.

The questioning of universality was accompanied by recent detailed studies of scale interactions in MHD turbulence. Many of the studies considered the so called shell-to-shell transfer functions and partial energy fluxes, either in numerical simulations, observations, closures, or from the theoretical point of view. In the following sections we give a review of the results in this area, considering the several regimes studied by different authors, and also some examples of possible sources of non-locality in MHD. Finally, we discuss the results in the context of universality and of phenomenological theories for MHD. To briefly summarize the results, several authors have shown that the locality of energy transfer is in question in MHD flows. In particular, it was shown from simulations that the transfer of energy in MHD has two components: a local one that shares similar properties with hydrodynamic turbulence, and a component coupling the velocity and magnetic fields for which energy from the large scales can be under some circumstances injected directly into the small scales without the intervention of 
intermediate scales.

\section{Indirect evidence of non-locality}

Some theoretical, phenomenological, and (more recently) numerical results indicate scale interactions in MHD can be, under some conditions, of a different nature than in hydrodynamic turbulence. In this section we review early theoretical indications of non-locality in MHD turbulence, as well as numerical results that support the theoretical arguments without directly measuring scale interactions.

Early studies of dynamo action, and of magnetic field evolution under flows with simple strain, show that a large scale flow can excite, through field line stretching, magnetic fields at widely separated scales. One of the first works along these lines is the work of Batchelor (1950) where he considered the similarity between the induction equation and the vorticity equation $(\boldsymbol{\omega}=\nabla \times \mathbf{u})$ :

$$
\frac{\partial \boldsymbol{\omega}}{\partial t}+\mathbf{u} \cdot \nabla \boldsymbol{\omega}=\boldsymbol{\omega} \cdot \nabla \mathbf{u}+\nu \nabla^{2} \omega
$$

While the second term on the l.h.s. advects the vorticity, the first term on the right (in three dimensions) produces vorticity by vortex stretching. For $P_{M}=\eta / \nu>1$ (the magnetic Prandtl number) Batchelor then concluded that the magnetic field would grow as magnetic field line stretching overcomes Ohmic dissipation. Later works considering stretching by uniform straining motion (Moffatt \& Saffman 1964, Zel'Dovich et al. 1984) showed that a large scale mag-

netic field can directly create small-scale magnetic fields. The work of Kazanstev (1968) considered a similar process under a random velocity field and described a nonlocal coupling that sustains the so-called small-scale dynamo, where magnetic fields are amplified at scales smaller than the integral scale of the flow. Several 
numerical simulations support these results, and show that smooth motions at the viscous scale give exponential growth of magnetic fields that can peak at the magnetic diffusion scale (Schekochihin et al. 2002a, 2004, 2002b).

The opposite limit, when the magnetic Prandtl number is much smaller than unity (a case of interest for industrial flows), is sometimes studied using the quasistatic approximation (see Knaepen \& Moreau 2008 for a review). In this case, an external uniform magnetic field is applied, and the magnetic Reynolds number is chosen small enough that magnetic field fluctuations are rapidly damped. In that limit the Lorentz force in the momentum equation reduces to linear Joule damping

$$
\frac{\partial \mathbf{u}}{\partial t}+\mathbf{u} \cdot \nabla \mathbf{u}=-\nabla p+\sigma B_{0}^{2} \nabla^{-2} \frac{\partial^{2} \mathbf{u}}{\partial z^{2}}+\nu \nabla^{2} \mathbf{u}
$$

where $\sigma$ is the conductivity of the medium and the uniform magnetic field $B_{0}$ is in the $z$ direction. The Joule damping, although anisotropic in spectral space, is roughly independent of the wavenumber, and unlike viscous damping is not concentrated at small scales but rather acts at all scales. As a result, the large-scale magnetic field in this approximation exerts work over all scales in the velocity field (damping turbulent fluctuations) in a non-local way. We will see that the shell-to-shell transfers indicate in some cases similar behavior of the Lorentz force even in cases far from this approximation.

Another important example concerns Alfvén waves, which are also non-linear solutions of the ideal MHD equations. Alfvénic states with $\mathbf{u}= \pm \mathbf{b}$ make the non-linear terms in Eqs. (11) and (2) zero, leaving only interactions with the large scale fields to transport energy across scales. Finally, it is worth mentioning here some recent attempts to build shell models of MHD turbulence (see e.g., Lessinnes et al. 2009, Plunian \& Stepanov 2007, Stepanov \& Plunian 2008). In 
these models, it was found that many features of steady state MHD turbulence can be reproduced using local coupling between shells, but that to reproduce the small-scale dynamo and turbulence at $P_{M} \gg 1$, non-local transfers have to be considered (Stepanov \& Plunian 2008).

\section{The shell-to-shell transfer}

In recent years, the increase in computing power allowed numerical exploration of MHD turbulence in different regimes. The development of shell-to-shell transfers (see Alexakis et al. 2005b, Dar et al. 2001, Debliquy et al. 2005) allowed for explicit computation of detailed scale interactions in MHD turbulence using the output stemming from the simulations and without the need to compute the more expensive triadic interactions. In this section we briefly introduce the isotropic shell-to-shell energy transfer functions, and describe how fluxes can be obtained from them.

A shell filter decomposition of the two fields is introduced as

$$
\begin{aligned}
& \mathbf{u}(\mathbf{x})=\sum_{K} \mathbf{u}_{K}(\mathbf{x}), \\
& \mathbf{b}(\mathbf{x})=\sum_{K} \mathbf{b}_{K}(\mathbf{x}),
\end{aligned}
$$

where

$$
\mathbf{u}_{K}(\mathbf{x})=\sum_{K_{1}<|\mathbf{k}| \leq K_{2}} \tilde{\mathbf{u}}(\mathbf{k}) e^{i \mathbf{k} \cdot \mathbf{x}}
$$

and

$$
\mathbf{b}_{K}(\mathbf{x})=\sum_{K_{1}<|\mathbf{k}| \leq K_{2}} \tilde{\mathbf{b}}(\mathbf{k}) e^{i \mathbf{k} \cdot \mathbf{x}}
$$

Here $\tilde{\mathbf{u}}(\mathbf{k})$ and $\tilde{\mathbf{b}}(\mathbf{k})$ are respectively the Fourier transforms of the velocity and magnetic fields with wavenumber $\mathbf{k}$. The shell filtered fields $\mathbf{u}_{K}$ and $\mathbf{b}_{K}$ are therefore defined as the field components whose Fourier transforms contain only 
wavenumbers in a given shell $K$. These shells can be defined with linear binning using $K_{1}=K$ and $K_{2}=K+1$, or alternatively with logarithmic binning using $K_{1}=\gamma^{n} K_{0}$ and $K_{2}=\gamma^{n+1} K_{0}$ for some positive $\gamma>1$ and integer $n(\gamma=2$ is often used). The latter definition has the advantage of being conceptually closer to the idea of "scale" of eddies in turbulence, which in general implies logarithmic division of wavenumbers. The former has the advantage of having a direct association with Alfvén waves, which are of the form $\mathbf{u}= \pm \mathbf{b} \sim e^{i(\mathbf{k} \cdot \mathbf{x} \pm \omega t)}$ in periodic boxes or in infinite domains, and which are more akin to linear treatment of spectral space. Note that the transfer among logarithmic shells can be reconstructed by summing over the linearly spaced shells.

Another variant when defining the shell filter decomposition has to do with the choice of using sharp filters (as in the equations above) or smooth filters (Evink 1994, 2005). This issue has raised some controversy in the hydrodynamic case, with claims that non-localities observed in simulations may be due to the commonly used sharp filters. Recent numerical comparisons (Domaradzki \& Carati 2007a, b have shown that results are only weakly dependent on the shape of the filter used, except in the case where a very broad smooth filter is considered. Moreover, recent theoretical results were able to show locality of hydrodynamic turbulence in Fourier space in the limit of infinite Reynolds numbers for both smooth and sharp filters (Aluie \& Eyink 2009a, Eyink \& Aluie 2009).

Based on the shell filter decomposition, the evolution of the kinetic energy in a shell $K, E_{u}(K)=\int \mathbf{u}_{K}^{2} / 2 d x^{3}$, can be derived from Eq. (11) as

$$
\frac{\partial E_{u}(K)}{\partial t}=\sum_{Q}\left[T_{u u}(Q, K)+T_{b u}(Q, K)\right]-\nu D_{u}(K)
$$


and for the magnetic energy, $E_{b}(K)=\int \mathbf{b}_{K}^{2} / 2 d x^{3}$, from Eq. (2)

$$
\frac{\partial E_{b}(K)}{\partial t}=\sum_{Q}\left[T_{b b}(Q, K)+T_{u b}(Q, K)\right]-\eta D_{b}(K),
$$

where the functions $D_{u}(K)$ and $D_{b}(K)$ express respectively the kinetic and magnetic energy dissipation in the shell $K$. The transfer functions $T_{u u}(Q, K)$, $T_{u b}(Q, K), T_{b b}(Q, K)$, and $T_{b u}(Q, K)$, that express the energy transfer between different fields and shells are given by

$$
\begin{aligned}
T_{u u}(Q, K) & =-\int \mathbf{u}_{K}(\mathbf{u} \cdot \nabla) \mathbf{u}_{Q} d x^{3}, \\
T_{b u}(Q, K) & =\int \mathbf{u}_{K}(\mathbf{b} \cdot \nabla) \mathbf{b}_{Q} d x^{3} \\
T_{b b}(Q, K) & =-\int \mathbf{b}_{K}(\mathbf{u} \cdot \nabla) \mathbf{b}_{Q} d x^{3} \\
T_{u b}(Q, K) & =\int \mathbf{b}_{K}(\mathbf{b} \cdot \nabla) \mathbf{u}_{Q} d x^{3}
\end{aligned}
$$

The function $T_{u u}(Q, K)$ measures the transfer rate of kinetic energy in the shell $Q$ to kinetic energy in the shell $K$ due to the advection term in the momentum equation (11). This is the non-linear transfer that is also present in hydrodynamic turbulence. Similarly, $T_{b b}(Q, K)$ expresses the rate of magnetic energy transferred from the shell $Q$ to magnetic energy in the shell $K$ due to the magnetic advection term. The Lorentz force is responsible for the transfer of energy from the magnetic field in the shell $Q$ to the velocity field in the shell $K$, as measured by $T_{b u}(Q, K)$. Finally, the term responsible for the stretching of magnetic field lines, the first term on the r.h.s. of Eq. (2), results in the transfer of kinetic energy from shell $Q$ to magnetic energy in shell $K$ and is expressed by $T_{u b}(Q, K)$. This is the term that describes magnetic induction and dynamo action.

All these transfer functions satisfy

$$
T_{v w}(Q, K)=-T_{w v}(K, Q)
$$


(where the subindices $v$ and $w$ stand for $u$ or $b$ ). This expression indicates that the rate at which the shell $Q$ gives energy to the shell $K$ is equal to the rate the shell $K$ receives energy from the shell $Q$, and is a necessary condition to define shellto-shell transfers that satisfy a detailed energy balance between shells. Then, the contribution of these transfers to the total energy flux can be computed as:

$$
\Pi_{v w}(k)=-\sum_{K=0}^{k} \sum_{Q} T_{v w}(K, Q) .
$$

Besides the total energy, the MHD equations have two more ideal invariants: the cross-helicity $C=\int \mathbf{u} \cdot \mathbf{b} d x^{3}$, and the magnetic helicity $H=\int \mathbf{a} \cdot \mathbf{b} d x^{3}$ where $\mathbf{a}$ is the vector potential such as $\mathbf{b}=\nabla \times \mathbf{a}$. These quantities also satisfy detailed balance equations equivalent to Eqs. (13) and (14). Shell-to-shell transfer functions for the magnetic helicity have been defined in Alexakis et al. (2006). Its transfer from shell $Q$ to shell $K$ is measured by

$$
T_{H}(K, Q)=\int \mathbf{b}_{K} \cdot\left(\mathbf{u}_{K} \times \mathbf{b}_{Q}\right) d x^{3} .
$$

The energy transfer functions were also generalized in recent works to consider the flux of energy in terms of the Elsässer variables (Alexakis et al. 2007a, 2005b, Carati et al. 2006), anisotropic transfers (Alexakis et al. 2007a,, Teaca et al. 2009), forward and backward transfers in an attempt to quantify backscatter required for subgrid models (Carati et al. 2006, Debliquv et al. 2005), and extensions to consider compressibility effects Graham et al. (2010), and kinetic plasma effects as in two-fluid MHD approximations (Mininni et al. 2007).

\section{Direct studies of multi-scale interactions}

The shell-to-shell energy transfers have been studied extensively (Alexakis et al. 2005b,

Carati et al. 2006, Dar et al. 2001, Debliquy et al. 2005, Mininni et al. 2005a, Verma 2004) 
for a variety of mechanically forced and decaying MHD flows in two and three dimensions. Depending on the configuration, different degrees of non-locality were reported. In the following subsections we present a short summary of the results discriminating by the forcing configuration. Overall, we can say that in all cases examined in the literature the transfers $T_{u u}$ and $T_{b b}$ have a local behavior: energy is transferred forward between nearby shells, in a fashion similar to what is observed in hydrodynamic turbulence (Alexakis et al. 2005a, Domaradzki \& Rogallo 1990, Mininni et al. 2006, Ohkitani \& Kida 1992, Yeung et al. 1995,

Zhou 1993). On the other hand, the $T_{b u}$ and $T_{u b}$ transfers that express the energy exchange between the velocity and the magnetic field have a rather different behavior.

\subsection{Forced isotropic and homogeneous turbulence}

As mentioned before, forced simulations of MHD turbulence can be attained by forcing both fields, or by forcing only the velocity field (in which case magnetic fields are sustained by dynamo action; a distinction must be done then between the kinematic regime, where the magnetic field has no backreaction on the flow, and the turbulent steady state where the magnetic field modifies the flow through the Lorentz force). The mechanically forced case is of more interest as it is closer to astrophysical and geophysical configurations, and as it is consistent with the constraint of magnetic flux conservation. The first studies of shell-to-shell transfer from simulations in such configuration were presented in Alexakis et al. (2005b). In the simulations with a resolution of $256^{3}$ grid points, the velocity field was forced with time independent mechanical forcing until a hydrodynamic turbulent steady state was reached. Two different forcing functions were studied: one 
non-helical and one helical. Magnetic Prandtl numbers of unity and smaller were considered. Once a hydrodynamic steady state was reached for each forcing function, a small magnetic field was introduced and, after the transient kinematic dynamo amplification, the system reached a steady state MHD turbulent regime. In such a state the transfer functions described in the previous section were computed. Typical results are illustrated in Fig. 1,

The $T_{u u}$ and $T_{b b}$ transfers were observed to behave in a similar fashion, giving direct and local transfer of energy. In Fig. 1, this is indicated by the negative and positive peaks, which show energy is removed by these transfer functions from smaller wavenumbers and given to slightly larger wavenumbers. However, for $T_{u b}$ a distinct behavior appeared. The large scale flow injected energy (through stretching) directly into the magnetic field at all scales. This manifests itself as a peak at the mechanical forcing scale for all receiving shells, and as an extended positive plateau (note positive $T_{u b}$ indicates energy given by the velocity field at shell $Q$ to magnetic field at shell $K$ ). In other words, at a given shell $K$, the magnetic field receives energy from the velocity field in all shells $Q<K$, and gives energy to the velocity field in shells $Q>K$. This result, reminiscent of the theoretical arguments by Batchelor (1950) and Zel'Dovich et al. (1984), was interpreted as the sustainment of the magnetic field against Ohmic dissipation by dynamo action: to maintain the magnetic field when only the velocity field is stirred, a nonzero flux from the velocity field to the magnetic field is required at all times. It is worth pointing out here that in the steady state this non-local transfer is small compared with the local transfers (approximately $10-20 \%$ at the resolutions studied). When considering Elsässer variables, the transfer functions were observed to become more local. 
The case of random forcing with magnetic Prandtl number of unity was studied in Carati et al. (2006) using $512^{3}$ simulations. The analysis, which used logarithmic binning, confirmed the previous results, showing local transfer in $T_{u u}$ and $T_{b b}$, and non-local coupling between the velocity and the magnetic field. This indicates that the phenomenon may be independent of the type of forcing, and associated to the stretching process that sustains the magnetic field. The work also discussed the possibility of splitting the transfer functions to discriminate between forward and backward contributions, which were used to discuss implications of the shell-to-shell transfers for LES models. Similar results were obtained for forced two dimensional MHD turbulence (Dar et al. 2001).

A different approach was considered by Yousef et al. (2007), who studied the steady state of small-scale dynamo action for $P_{M} \leq 1$. Instead of using transfer functions, to measure the different components of the energy flux they considered the Politano-Pouquet relations (6) in terms of the velocity and the magnetic field $\left\langle\delta u_{\|}\left(|\delta \mathbf{u}|^{2}+|\delta \mathbf{b}|^{2}\right)\right\rangle \mp\left\langle\delta b_{\|}\left(|\delta \mathbf{u}|^{2}+|\delta \mathbf{b}|^{2}\right)\right\rangle \pm 2\left\langle\delta \mathbf{u} \cdot \delta \mathbf{b}\left(\delta u_{\|} \mp \delta b_{\|}\right)\right\rangle=-\frac{4}{3} \epsilon^{ \pm} l$

together with Chandrasekhar (1951) law

$$
\left\langle\delta u_{\|}^{3}\right\rangle-6\left\langle b_{\|}^{2} \delta u_{\|}\right\rangle=-\frac{4}{5} \epsilon l
$$

where $\epsilon$ is the total energy flux. The authors discriminated between the different terms to look how they balanced to give rise to the direct flux. Each of the terms in these expressions can indeed be associated to the counterpart in real space of the $\Pi_{u u}, \Pi_{b b}$, and $\Pi_{u b}+\Pi_{b u}$ fluxes in Fourier space.

The dominant balance was identified between $(4 / 5) \epsilon l$ and $6\left\langle b_{\|}^{2} \delta u_{\|}\right\rangle$, and they concluded that at their available resolution, the local direct cascade of energy was 
"short-circuited" by the transfer of kinetic energy into magnetic energy. They also associated this non-local coupling with the folded structure of the small-scale magnetic field. Using the shell-to-shell transfer approach, Alexakis et al. (2007a) further showed that the non-local effects disappear if phases are randomized for the two fields, which also make the current sheet and folded structures disappear.

The non-local effects play a more important role in the kinematic dynamo regime (Mininni et al. 2005a), as in that case the turbulence is not in a steady regime and $T_{u b}$ accounts for all mechanisms that amplify the magnetic field. In that case, the $T_{u b}$ transfer has been shown to be useful to identify and quantify scale-by-scale sources of dynamo action (Alexakis et al. 2007b, Mininni et al. 2005a).

\subsection{Freely decaying turbulence}

The non-local effects observed in forced turbulence are either absent or negligible in the freely decaying case. In Debliquy et al. (2005), $512^{3}$ simulations of freely decaying MHD turbulence were considered. The $T_{u u}$ and $T_{b b}$ transfers are similar to the forced case (see Fig. 2) and indicate local direct transfer. However, the $T_{u b}$ and $T_{b u}$ transfer functions were also observed to be local, with most of the transfer between the velocity and the magnetic field taking place between the same shell. The remaining transfer (for non-neighboring shells) was observed to decay more slowly than in the $T_{u u}$ and $T_{b b}$ functions, but except for this detail no other indications of non-locality were reported.

Similar results were obtained from analysis of solar wind turbulence (Strumik \& Macek 20 Solar wind turbulence is often considered the MHD equivalent of hydrodynamic freely decaying wind tunnel turbulence (see Bruno \& Carbone 2005 for a review). From 1996 Ulysses magnetometers time series and using a Markov process ap- 
proach, Strumik \& Macek (2008b) concluded that the transfer of magnetic to magnetic energy was local. Then, using velocity and magnetic field time series from ACE spacecraft from 1999 to 2006 and performing the same analysis on the remaining transfers they concluded that all transfers were local.

The differences between the forced and freely decaying cases can be understood as in the mechanically forced runs, the velocity field has to continuously supply energy to the magnetic field in order to sustain it against Ohmic dissipation. This is not necessarily the case for freely decaying runs where both fields are dissipated in time.

\subsection{Anisotropic turbulence}

Recently, the shell-to-shell transfers were extended to consider anisotropies when an external uniform magnetic field is imposed. This case is of interest as in many astrophysical problems a strong large-scale magnetic field is present creating small-scale anisotropy. Unlike hydrodynamic turbulence, MHD turbulence does not recover isotropy at small scales, and theoretical and numerical results indicate anisotropy becomes stronger at smaller scales.

To study anisotropic transfers, different foldings of the shells in Fourier space

can be implemented. Fig 3 shows the possible options. In Alexakis et al. (2007a), anisotropic shell-to-shell transfer functions were introduced folding Fourier shells in cylinders (associated to wavenumbers $k_{\perp}$ perpendicular to the mean magnetic field) and in planes (associated to parallel wavenumbers $k_{\|}$). Shell-to-shell transfers were only considered for the Elsässer variables, but the fluxes were reconstructed from these functions to measure the relative contribution of nonlocality to the total flux. Freely decaying simulations with spatial resolution of 
$256^{3}$ grid points were analyzed, and the amplitude of the imposed magnetic field was varied from 0 to 15 (in units of the initial small scale fluctuations). The transfer functions of the two Elsässer energies were found local in both parallel and perpendicular directions, irrespectively of the amplitude of the external field. However, interactions between the counterpropagating Alfvén waves were reported to become non-local. For strong magnetic fields, most of the energy flux in the perpendicular direction was found to be due to interactions with modes with $k_{\|}=0$ (see Fig. 4). In the parallel direction, however, $k_{\|}=0$ modes cannot transfer energy and most of the interactions were observed to take place with modes near $k_{\|} \approx 0$. The results are in qualitative agreement with predictions from weak turbulence theory (Galtier et al. 2000) and with recent non-local phenomenological models (Alexakis 2007).

A different approach to study anisotropic transfers was presented by Teaca et al. (2009), who decomposed the spectral space into rings, studying then transfers along radial and angular directions in spectral space (which they termed "ring-to-ring" transfers). They considered forced simulations of MHD turbulence with an imposed magnetic field, with a spatial resolution of $512^{3}$ grid points and varying the imposed magnetic field between 0 to $\sqrt{10}$ (in units of the small-scale magnetic field fluctuations). They also observed dominance of energy transfer in the direction perpendicular to the uniform magnetic field, and suppression of the transfer in the parallel direction. Their approach is useful to understand how energy is angularly distributed in spectral space to create anisotropy. Non-local effects with the forcing shell were observed in the shell-to-shell transfers, but in the angular ring-to-ring transfers were too weak to be noticed. 


\subsection{Magnetic helicity and the inverse cascade}

Non-local transfers were also reported in investigations of the cascade of magnetic helicity. Magnetic helicity is an ideal invariant in MHD, that is known to cascade inversely (to the large scales) in a turbulent flow (Alexakis et al. 2006, Brandenburg 2001, Brandenburg \& Subramanian 2005, Gómez \& Mininni 2004,

Meneguzzi et al. 1981, Pouquet et al. 1976). The generation of large scale magnetic fields in galaxies and other astrophysical bodies is sometimes attributed to this inverse cascade. In Alexakis et al. (2006) magnetically and mechanically forced simulations were considered. In both cases, both local and non-local transfers were observed. At early times, magnetic helicity was observed to cascade inversely and locally from the closest neighbor shells, and non-locally from the forced shells. When the correlation length became of the size of the box, the direct input from the forced scales became dominant, and a local direct transfer of helicity from large to small scales also developed. This latter effect was speculated to be dependent on boundary conditions and therefore non-universal.

In the mechanically forced case the inverse cascade of helicity was associated to the large-scale dynamo $\alpha$-effect (Brandenburg 2001, Brandenburg \& Subramanian 2005, Krause \& Raedler 1980, Pouquet et al. 1976, Steenbeck et al. 1966). In that case, the mechanical forcing creates equal amounts of magnetic helicity of opposite signs at large and small scales. The process can be understood using the conceptual "stretch, twist, and fold" (STF) dynamo mechanism (Childress \& Gilbert 1995, Vainshtein \& Zeldovich 1972). Each time a closed magnetic flux tube is twisted by the helical velocity field, magnetic helicity is created at large scales, while small scale magnetic field lines are twisted in the opposite direction thus creating equal amount of magnetic helicity of opposite sign in the small scales. As the 
STF process is repeated, the large-scale helicity is transferred inversely both locally and non-locally (with constant flux), while the small-scale helicity is pushed towards smaller scales (see Fig 5). This latter process removes magnetic helicity from the large scales and allows the magnetic field to "disentangle" through reconnection events, destroying in that way magnetic helicity (Alexakis et al. 2006, 2007b). At this moment it is unclear whether this processes should be associated to a cascade (i.e., if the process takes place with constant flux), although results in Alexakis et al. (2007b) and Mininni \& Pouquet (2009) suggest this may not be the case.

\section{Non-local interactions and universality of MHD turbulence}

The above considerations led several authors to consider whether some of the usual assumptions in hydrodynamic turbulence hold in the MHD case. From the shell-to-shell transfer, the scenario pictured in Fig. 6] seems to arise for the energy: interactions between the same fields are mostly local, and interactions between the velocity and the magnetic field can have different degrees of non-locality depending on whether the turbulence is forced or freely decaying, depending on how the velocity and the magnetic fields are maintained against dissipation in the forced case, and depending on the presence of an external magnetic field. It is unclear for the moment whether the varying degree of non-locality with the configuration will converge to a universal solution for very large Reynolds numbers.

Theoretical arguments considering interactions in MHD turbulence also obtained conflicting results. Using the EDQNM closure, Pouquet et al. (1976) re-

ported non-local interactions which were associated to Alfvén waves. In Verma (2003, 
Scalp Interactions in MHD Turbulence

2004) and Verma et al. (2005), field-theoretic calculations were used to compute the shell-to-shell transfers and it was concluded that they were local except for the transfer between the velocity and the magnetic field, which was found to be somewhat non-local. The helicity transfer was also found to be non-local. Recently, Aluie \& Evink (2009b) gave strict bounds for fluxes in MHD turbulence under the assumptions that both the velocity and the magnetic energy follow power laws in the inertial range between $k^{-1}$ and $k^{-3}$. The velocity-tovelocity and magnetic-to-magnetic fluxes were found to be local in the limit of infinite Reynolds number, and the fluxes coupling velocity and magnetic fields were found to be local although counterexamples to their proof as the ones mentioned in Sect. 2 were acknowledged. However, these results shed light into why some simulations were found to be more local than others, as mechanisms as the small-scale dynamo can be expected to be less relevant in freely decaying turbulence in approximate equipartition between the two fields.

At presently attainable spatial resolutions, other indications of possible nonuniversal behavior has been reported in numerical simulations. In Dmitruk et al. (2003), simulations of forced reduced magnetohydrodynamics (RMHD) where presented where the energy spectrum changed its power law depending on the timescale of the external forcing. Spectra compatible with Kolmogorov, Iroshnikov-Kraichnan, weak turbulence theory, or even steeper laws were observed. The RMHD equations correspond to an approximation of the MHD equations when a strong external magnetic field is imposed. Similar results were reported by Mason et al. (2008), who considered forced MHD with an imposed magnetic field. Other numerical simulations of forced MHD turbulence (see e.g., Beresnyak \& Lazarian 2009, Haugen et al. 2003, Müller et al. 2003, Müller \& Grappin 2005) also reported con- 
flicting results. In freely decaying isotropic turbulence, some simulations were observed to develop Iroshnikov-Kraichnan scaling while others Kolmogorov-like scaling (Mininni \& Pouquet 2007, 2009, Müller \& Grappin 2005). Recently, large resolution simulations of freely decaying MHD flows showed that depending on the amplitude of the dynamically consistent large-scale magnetic field, different power laws can be realized (Lee et al. 2009). Finally, recent studies of spectral laws in solar wind data (Podesta et al. 2007) indicate that many of these power laws can also be identified in space plasmas.

Although the main aim of this review is to consider studies of scale interactions in MHD, in this context it is worth mentioning some of the existing phenomenological theories for MHD turbulence. While Iroshnikov and Kraichnan considered small scale fluctuations as isotropic, it is clear now that MHD turbulence does not recover isotropy at small scales (Goldreich \& Sridhar 1995), Milano et al. 2001, Shebalin et al. 1983) and may become even more anisotropic as the scales are decreased. To take this into account, a different MHD spectrum has been advocated in Goldreich \& Sridhar (1995), whereby the anisotropy of the flow induces a Kolmogorov-like spectrum in the perpendicular direction. A balance between linear and non-linear timescales (the Alfvén and turnover times) is assumed which leads to a "critical balance" of the form $k_{\|} B_{0} \sim k_{\perp} b_{l}$. Another anisotropic model based on dynamic alignment of the velocity and magnetic fields (Boldyrev 2006) gives IK-like scaling in the perpendicular direction. In this case, the angle between the two fields decreases (and therefore the fields become more aligned) with the scale as $\sim l^{1 / 4}$. Consideration of this alignment in the Politano-Pouquet relations leads to the aforementioned scaling for the energy spectrum. Early extensions to flows with sizable cross-correlations can be found in 
Galtier et al. (2000), Grappin et al. (1983). Other models have considered transitions from Kolmogorov to Iroshnikov-Kraichnan scaling by taking different combinations of the non-linear and Alfvén timescales (Matthaeus \& Zhou 1989), or consider non-locality (Alexakis 2007).

Therefore, while the assumptions of locality and of isotropization of the small scales common in hydrodynamic turbulence allow for a simpler phenomenological treatment of MHD, the development of local anisotropies, the variety of time scales in the problem (see Zhou et al. 2004 for a review), and the different simulations showing scaling consistent with different phenomenological theories, led some authors to discuss some of these assumptions. In Schekochihin et al. (2008), non-locality, anisotropy, and non-universality were considered as defining properties of MHD turbulence. The authors argued that the small-scale dynamo, a fundamental process in MHD turbulence, shows clear signatures of non-locality (Haugen et al. 2003, 2004, Mininni et al. 2005a, Schekochihin et al. 2002a, 2004, $2002 \mathrm{~b})$. They also argued that anisotropy is intrinsic to MHD, and that nonuniversality manifests itself just from the needed distinction between MHD turbulence in the presence and in the absence of a strong mean field. Similar concerns about universal behavior in MHD were discussed in Lee et al. (2009) for the case of freely decaying turbulence. Beresnyak \& Lazarian (2009) consider the lack of a "bottleneck" in MHD (an accumulation of energy at the beginning of the viscous range observed in hydrodynamic turbulence) as evidence of non-locality (see also Graham et al. 2009). In some sense, some of these discussions can be tracked back to early considerations of freely decaying MHD turbulence and the processes of selective decay (Kinnev et al. 1995, Matthaeus \& Montgomery 1980, Mininni et al. 2005b, Ting et al. 1986) and dy- 
namic alignment (Ghosh et al. 1988, Grappin et al. 1983, Mininni et al. 2005c, Pouquet et al. 1986). MHD, having three ideal invariants, is known to decay for very long times into different attractors depending on the initial ratio of these invariants (Stribling \& Matthaeus 1991, Ting et al. 1986). Although these solutions involve final stages of the decay, recent numerical simulations showed that these relaxed states can be realized locally in the flow in very short times scales (Mason et al. 2006, Matthaeus et al. 2008, Perez \& Boldyrev 2009, Servidio et al. 2008), giving rise to different regimes.

\section{Concluding remarks}

Since the success of the phenomenological theory of Kolmogorov in hydrodynamic turbulence, several attempts have been made to apply similar considerations to tackle MHD turbulence. The presence of waves and of several time scales, and of several ideal invariants limited these approaches giving rise to many possible models. Solar wind observations and numerical simulations later showed that assumptions like isotropy of the small scales, or equipartition between the fields, may not hold in the MHD case. More recently, the increase in computing power allowed for some exploration of the parameter space giving rise to conflicting results for scaling laws in the energy spectrum.

The recent introduction of shell-to-shell transfers allowed for detailed studies of scale interactions in MHD turbulence and opened the door to discuss another hypothesis: that of locality of interactions between scales. The results, at intermediate spatial resolutions and Reynolds numbers, show different degrees of non-locality depending on the configuration studied: forced or freely decaying turbulence, in presence or in absence of an external magnetic field, etc. Non- 
local transfers, when observed, involve the coupling between the velocity and the magnetic field, or the transfer of magnetic helicity. In the former case, the nonlocal transfers were not larger than $10-20 \%$ of the total, although they played fundamental roles, e.g., sustaining the magnetic field by dynamo action against Ohmic dissipation.

In spite of some conflicting results in the simulations and theory, there is growing consensus that MHD turbulence is less local than hydrodynamic turbulence. To what extent it is a matter of debate. It is unclear for the moment whether these effects will go away for larger Reynolds numbers, or if they stay how much impact they will have in the flow dynamics, and under what conditions. However, the different degrees of non-locality observed at present resolutions, and the existence of non-local processes in MHD (as, e.g., the small scale dynamo), call for a discussion about the validity of the hypothesis of locality of interactions, and of whether there is only one kind of MHD turbulence or many. This raises the question of what is the definition of MHD turbulence in phenomenological or theoretical approaches. If only configurations as the ones in the solar wind (with an imposed magnetic field) are to be considered, then a universal scaling (or several classes of universality) may be possibly identified. However, if processes like the small-scale dynamo, the large-scale dynamo, and inverse cascades are to be considered as manifestations of MHD turbulence, non-local interactions and non-universal behavior may persist even for very large Reynolds numbers. In this context, many of the works reviewed here may have to be revisited in the following years, as experiments and increased computing power will allow us to explore new regions of the parameter space of MHD turbulence. 


\section{$7 \quad$ Summary points}

1. Simply applying properties of hydrodynamic turbulence to the MHD case may not be possible. In particular, assumptions of scale locality of MHD turbulence must be tested in experiments and simulations.

2. Shell-to-shell transfer functions allow for detailed studies of coupling between fields and scales in numerical simulations. The shell-to-shell transfers can also be associated with physical processes such as as Alfvén wave interactions, Joule damping, and dynamo action.

3. The degree of non-locality observed at the presently attainable spatial resolutions depends on the configuration.

4. Mechanically forced turbulence shows local transfer of magnetic and kinetic energy, but the coupling between the velocity and magnetic field that sustains the latter against Ohmic dissipation is non-local.

5. In freely decaying MHD turbulence, non-local effects seem to be negligible.

6. Studies of the energy transfer in the presence of an imposed magnetic field show most of the transfer takes place in the direction perpendicular to the external field, with strong non-local interactions with modes with $k_{\|}=0$.

7. The transfer of energy for the Elsässer variables is more local than the transfer in terms of the velocity and magnetic fields.

8. The shell-to-shell transfer of magnetic helicity is more complex, with superimposed direct and inverse transfers. The inverse transfer has a local component, and a non-local one that moves energy from the forced scale directly to the largest scales in the system. 


\section{Acknowledgments}

The National Center for Atmospheric Research is sponsored by the National

Science Foundation. The author acknowledges fruitful discussions with Alexandros Alexakis, Daniele Carati, Gregory Eyink, Annick Pouquet, and Alexander Schekochihin, and figures gently provided by A. Alexakis and D. Carati. The author also expresses his gratitude to A. Pouquet for her careful reading of the manuscript. PDM is a member of the Carrera del Investigador Científico of CONICET, and acknowledges support from grants UBACYT X468/08 and PICT 2007-02211.

\section{References}

1. Alexakis A. 2007. Nonlocal phenomenology for anisotropic magnetohydrodynamic turbulence. Astrophys. J. 667:L93-L96

2. Alexakis A, Bigot B, Politano H, Galtier S. 2007a. Anisotropic fluxes and nonlocal interactions in magnetohydrodynamic turbulence. Phys. Rev. E 76:056313

3. Alexakis A, Mininni PD, Pouquet A. 2005a. Imprint of large-scale flows on turbulence. Phys. Rev. Lett. 95:264503

4. Alexakis A, Mininni PD, Pouquet A. 2005b. Shell to shell energy transfer in MHD. I. Steady state turbulence. Phys. Rev. E 72:046301

5. Alexakis A, Mininni PD, Pouquet A. 2006. On the inverse cascade of magnetic helicity. Astrophys. J. 640:335-343

6. Alexakis A, Mininni PD, Pouquet A. 2007b. Turbulent cascades, transfer, and scale interactions in magnetohydrodynamics. New J. Phys. 9:298 
7. Aluie H, Eyink GL. 2009a. Localness of energy cascade in hydrodynamic turbulence. II. Sharp spectral filter. Phys. Fluids 21:115108

8. Aluie H, Eyink GL. 2009b. Scale-locality of magnetohydrodynamic turbulence. Phys. Rev. Lett., in press (arXiv:0912.3752).

9. Batchelor GK. 1950. On the spontaneous magnetic field in a conducting liquid in turbulent motion. Proc. Roy. Soc. of London Series A 201:405-416

10. Beresnyak A, Lazarian A. 2009. Comparison of spectral slopes of magnetohydrodynamic and hydrodynamic turbulence and measurements of alignment effects. Astrophys. J. 702:1190-1198

11. Boldyrev S. 2006. Spectrum of magnetohydrodynamic turbulence. Phys. Rev. E 96:115002

12. Brandenburg A. 2001. The inverse cascade and nonlinear alpha-effect in simulations of isotropic helical hydromagnetic turbulence. Astrophys. J. 550:824-840

13. Brandenburg A, Subramanian K. 2005. Astrophysical magnetic fields and nonlinear dynamo theory. Phys. Rep. 417:1-209

14. Bruno R, Carbone V. 2005. The solar wind as a turbulence laboratory. Liv. Rev. Solar Phys. 2:4

15. Carati D, Debliquy O, Knaepen B, Teaca B, Verma M. 2006. Energy transfers in forced MHD turbulence. J. of Turb. 7:51

16. Carlier J, Laval JP, Stanislas M. 2001. Some experimental support at a high Reynolds number to a new hypothesis for turbulence modeling. Compt. Rend. de l'Academ. des Sci. Ser. II 329:35-40

17. Chandrasekhar S. 1951. The invariant theory of isotropic turbulence in magnetohydrodynamics. Proc. R. Soc. London, Ser. A 204:435-449 
18. Childress S, Gilbert AD. 1995. Stretch, twist, fold: the fast dynamo. Berlin: Springer-Verlag

19. Dar G, Verma MK, Eswaran V. 2001. Energy transfer in two-dimensional magnetohydrodynamic turbulence: formalism and numerical results. Physica $D$ $157: 207-225$

20. Debliquy O, Verma MK, Carati D. 2005. Energy fluxes and shell-to-shell transfers in three-dimensional decaying magnetohydrodynamic turbulence. Phys. Plasmas 12:042309

21. Dmitruk P, Gómez DO, Matthaeus WH. 2003. Energy spectrum of turbulent fluctuations in boundary driven reduced magnetohydrodynamics. Phys. Plasmas $10: 3584-3591$

22. Domaradzki JA. 1988. Analysis of energy transfer in direct numerical simulations of isotropic turbulence. Phys. Fluids 31:2747-2749

23. Domaradzki JA, Carati D. 2007a. An analysis of the energy transfer and the locality of nonlinear interactions in turbulence. PoF 19:085112

24. Domaradzki JA, Carati D. 2007b. A comparison of spectral sharp and smooth filters in the analysis of nonlinear interactions and energy transfer in turbulence. PoF 19:085111

25. Domaradzki JA, Rogallo RS. 1990. Local energy transfer and nonlocal interactions in homogeneous, isotropic turbulence. Phys. Fluids 2:413-426

26. Eyink GL. 1994. Energy dissipation without viscosity in ideal hydrodynamics. i. fourier analysis and local energy transfer. Physica D 78:222-240

27. Eyink GL. 2005. Locality of turbulent cascades. Physica D 207:91-116 
28. Eyink GL, Aluie H. 2009. Localness of energy cascade in hydrodynamic turbulence. I. Smooth coarse graining. Phys. Fluids 21:115107

29. Galtier S, Nazarenko SV, Newell AC, Pouquet A. 2000. A weak turbulence theory for incompressible magnetohydrodynamics. J. Plasma Phys. 63:447-488

30. Galtier S, Pouquet A, Mangeney A. 2005. On spectral scaling laws for incompressible anisotropic magnetohydrodynamic turbulence. Phys. Plasmas 12:092310

31. Ghosh S, Matthaeus WH, , Montgomery DC. 1988. The evolution of cross helicity in driven/dissipative two-dimensional magnetohydrodynamics. Phys. Fluids $31: 2171-2184$

32. Goldreich P, Sridhar P. 1995. Toward a theory of interstellar turbulence. 2. Strong Alfvenic turbulence. Astrophys. J. 438:763-775

33. Gómez DO, Mininni PD. 2004. Direct numerical simulations of helical dynamo action: MHD and beyond. Nonlin. Proc. Geophys. 11:619-629

34. Gomez T, Politano H, Pouquet A. 1999. On the validity of a nonlocal approach for MHD turbulence. Phys. Fluids 11:2298-2306

35. Graham JP, Cameron R, Schuessler M. 2010. Turbulent small-scale dynamo action in solar surface simulations. Submitted to Astrophys. J. (arXiv:1002.2750).

36. Graham JP, Mininni PD, Pouquet A. 2009. Lagrangian-averaged model for magnetohydrodynamic turbulence and the absence of bottlenecks. Phys. Rev. E 80:016313

37. Grappin R, Pouquet A, Léorat J. 1983. Dependence on correlation of MHD turbulence spectra. Astron. Astrophys. 126:51-56

38. Haugen NEL, Brandenburg A, Dobler W. 2003. Is nonhelical hydromagnetic turbulence peaked at small scales? Astrophys. J. 597:L141-L144 
39. Haugen NEL, Brandenburg A, Dobler W. 2004. Simulations of nonhelical hydromagnetic turbulence. Phys. Rev. E 70:016308

40. Iroshnikov PS. 1963. Turbulence of a conducting fluid in a strong magnetic field. Sov. Astron. 7:566-571

41. Kazanstev AP. 1968. Enhancement of a magnetic field by a conducting fluid. Sov. Phys. JETP 26:1031-1034

42. Kinney R, McWilliams JC, Tajima T. 1995. Coherent structures and turbulent cascades in two-dimensional incompressible magnetohydrodynamic turbulence. Phys. Plasmas 2:3623-3639

43. Knaepen B, Moreau R. 2008. Magnetohydrodynamic turbulence at low magnetic Reynolds number. Ann. Rev. Fluid Mech. 40:25-45

44. Kraichnan RH. 1959. The structure of isotropic turbulence at very high reynolds numbers. J. Fluid Mech. 5:497-543

45. Kraichnan RH. 1965. Inertial-range spectrum of hydromagnetic turbulence. Phys. Fluids 8:1385-1387

46. Krause F, Raedler KH. 1980. Mean-field magnetohydrodynamics and dynamo theory. New York: Pergamon Press

47. Lee E, Brachet ME, Pouquet A, Mininni PD. 2009. Lack of universality in decaying magnetohydrodynamic turbulence. Phys. Rev. E 81:016318

48. Lessinnes T, Carati D, Verma MK. 2009. Energy transfers in shell models for magnetohydrodynamic turbulence. Phys. Rev. E 79:066307

49. Mason J, Cattaneo F, Boldyrev S. 2006. Dynamic alignment in driven magnetohydrodynamic turbulence. Phys. Rev. Lett. 97:255002 
50. Mason J, Cattaneo F, Boldyrev S. 2008. Numerical measurements of the spectrum in magnetohydrodynamic turbulence. Phys. Rev. E 77:036403

51. Matthaeus WH, Montgomery D. 1980. Selective decay hypothesis at high mechanical and magnetic reynolds numbers. Ann. N. Y. Acad. Sci. 357:203

52. Matthaeus WH, Pouquet A, Mininni PD, Dmitruk P, Breech B. 2008. Rapid alignment of velocity and magnetic field in magnetohydrodynamic turbulence. Phys. Rev. Lett. 100:085003

53. Matthaeus WH, Zhou Y. 1989. Extended inertial range phenomenology of magnetohydrodynamic turbulence. Phys. Fluids B 1:1929-1931

54. Meneguzzi M, Frisch U, Pouquet A. 1981. Helical and nonhelical turbulent dynamos. Phys. Rev. Lett. 47:1060-1064

55. Milano LJ, Matthaeus WH, Dmitruk P, Montgomery DC. 2001. Local anisotropy in incompressible magnetohydrodynamic turbulence. Phys. Plasmas 8:26732681

56. Mininni PD, Alexakis A, Pouquet A. 2005a. Shell to shell energy transfer in MHD. II. Kinematic dynamo. Phys. Rev. E 72:046302

57. Mininni PD, Alexakis A, Pouquet A. 2006. Large-scale flow effects, energy transfer, and self-similarity on turbulence. Phys. Rev. E 74:016303

58. Mininni PD, Alexakis A, Pouquet A. 2007. Energy transfer in Hall-MHD turbulence: cascades, backscatter, and dynamo action. J. Plasma Phys. 73:377-401

59. Mininni PD, Alexakis A, Pouquet A. 2008. Nonlocal interactions in hydrodynamic turbulence at high Reynolds numbers: The slow emergence of scaling laws. Phys. Rev. E 77:036306 
60. Mininni PD, Montgomery DC, Pouquet A. 2005b. Numerical solutions of the three-dimensional magnetohydrodynamic $\alpha$ model. Phys. Rev. E 71:046304

61. Mininni PD, Montgomery DC, Pouquet A. 2005c. A numerical study of the alpha model for two-dimensional magnetohydrodynamic turbulent flows. Phys. Fluids $17: 035112$

62. Mininni PD, Pouquet A. 2007. Energy spectra stemming from interactions of alfven waves and turbulent eddies. Phys. Rev. Lett. 99:254502

63. Mininni PD, Pouquet A. 2009. Finite dissipation and intermittency in magnetohydrodynamics. Phys. Rev. E 80:025401

64. Moffatt HK. 1978. Magnetic field generation in electrically conducting fluids. Cambridge: Cambridge Univ. Press

65. Moffatt HK, Saffman PG. 1964. Comment on "Growth of a weak magnetic field in a turbulent conducting fluid with large magnetic Prandtl number. Phys. Fluids 7:155

66. Monchaux R, Berhanu M, Bourgoin M, Moulin M, Odier P, et al. 2007. Generation of a magnetic field by dynamo action in a turbulent flow of liquid sodium. Phys. Rev. Lett. 98:044502

67. Müller WC, Biskamp D, Grappin R. 2003. Statistical anisotropy of magnetohydrodynamic turbulence. Phys. Rev. E 67:066302

68. Müller WC, Grappin R. 2005. Spectral energy dynamics in magnetohydrodynamic turbulence. Phys. Rev. Lett. 95:114502

69. Nazarenko SV, Newell AC, Galtier S. 2001. Non-local MHD turbulence. Physica D 152:646-652 
70. Ohkitani K, Kida S. 1992. Triad interactions in a forced turbulence. Phys. Fluids A 4:794-802

71. Perez JC, Boldyrev S. 2009. Role of cross-helicity in magnetohydrodynamic turbulence. Phys. Rev. Lett. 102:025003

72. Plunian F, Stepanov R. 2007. A non-local shell model of hydrodynamic and magnetohydrodinamic turbulence. New J. Phys. 9:294

73. Podesta JJ, Roberts DA, Goldstein ML. 2007. Spectral exponents of kinetic and magnetic energy spectra in solar wind turbulence. Astrophys. J. 664:543-548

74. Politano H, Pouquet A. 1998a. Dynamical length scales for turbulent magnetized flows. Geophys. Res. Lett. 25:273-276

75. Politano H, Pouquet A. 1998b. von kármán-howarth equation for magnetohydrodynamics and its consequences on third-order longitudinal structure and correlation functions. Phys. Rev. E 57:R21-R24

76. Poulain C, Mazellier N, Chevillard L, Gagne Y, Baudet C. 2006. Dyamics of spatial Fourier modes in turbulence. Sweeping effect, long-time correlations and temporal intermittency. Eur. Phys. J. B 53:219-224

77. Pouquet A, Frisch U, Léorat J. 1976. Strong MHD helical turbulence and the nonlinear dynamo effect. J. Fluid Mech. 77:321-354

78. Pouquet A, Meneguzzi M, , Frisch U. 1986. The growth of correlations in MHD turbulence. Phys. Rev. A 33:4266-4276

79. Schekochihin A, Cowley SC, Yousef TA. 2008. MHD turbulence: nonlocal, anisotropic, nonuniversal? In IUTAM Symposium on computational physics and new perspectives in turbulence, ed. Y Kaneda. Dordrecht, The Netherlands: Springer 
80. Schekochihin AA, Cowley SC, Hammett GW, Maron JL, McWilliams JC. 2002a.

A model of nonlinear evolution and saturation of the turbulent MHD dynamo. New J. Phys. 4:1-22

81. Schekochihin AA, Cowley SC, Taylor S, Maron JL, McWilliams JC. 2004. Simulations of the small-scale turbulent dynamo. Astrophys. J. 612:276-307

82. Schekochihin AA, Maron JL, Cowley SC, McWilliams JC. 2002b. The small-scale structure of magnetohydrodynamic turbulence with large magnetic Prandtl numbers. Astrophys. J. 576:806-813

83. Servidio S, Matthaeus WH, Dmitruk P. 2008. Depression of nonlinearity in decaying isotropic MHD turbulence. Phys. Rev. Lett. 100:095005

84. Shebalin JV, Matthaeus WH, Montgomery D. 1983. Anisotropy in MHD turbulence due to a mean magnetic field. J. Plasma Phys. 29:525-547

85. Shen X, Warhaft Z. 2000. The anisotropy of the small-scale structure in high reynolds number $\left(r_{\lambda} \sim 1000\right)$ turbulent shear flow. Phys. Fluids 12:2976-2989

86. Steenbeck M, Krause F, Rädler KH. 1966. Berechnung der mittleren LorentzFeldstaerke $\overline{\mathbf{v} \times \mathbf{b}}$ fuer ein elektrisch leitendendes Medium in turbulenter, durch Coriolis-Kraefte beeinflußter Bewegung. Z. Naturforsch. 21a:369-376

87. Stepanov R, Plunian F. 2008. Phenomenology of turbulent dynamo growth and saturation. Astrophys. J. 680:809-815

88. Stribling T, Matthaeus WH. 1991. Relaxation processes in a low-order threedimensional magnetohydrodynamics model. Phys. Fluids B 3:1848-1864

89. Strumik M, Macek WM. 2008a. Statistical analysis of transfer of fluctuations in solar wind turbulence. Nonlin, Processes Geophys. 15:607-613 
90. Strumik M, Macek WM. 2008b. Testing for markovian character and modeling of intermittency in solar wind turbulence. Phys. Rev. E 78:026414

91. Teaca B, Verma MK, Knaepen B, Carati D. 2009. Energy transfer in anisotropic magnetohydrodynamic turbulence. Phys. Rev. E 79:046312

92. Ting AC, Matthaeus WH, Montgomery D. 1986. Turbulent relaxation processes in magnetohydrodynamics. Phys. Fluids 29:3261-3274

93. Vainshtein SI, Zeldovich YB. 1972. Origin of magnetic fields in astrophysics. Sov. Phys. Usp. 15:159-172

94. Verma MK. 2003. Field theoretic calculation of energy cascade rates in non-helical magnetohydrodynamic turbulence. Pramana 61:577-594

95. Verma MK. 2004. Statistical theory of magnetohydrodynamic turbulence: recent results. Phys. Rep. 401:229-380

96. Verma MK, Ayyer A, Chandra AV. 2005. Energy transfers and locality in magnetohydrodynamic turbulence. Phys. Plasmas 12:082307

97. Wiltse JM, Glezer A. 1993. Manipulation of free shear flows using piezoelectric actuators. J. Fluid Mech. 249:261-285

98. Wiltse JM, Glezer A. 1998. Direct excitation of small-scale motions in free shear flows. Phys. Fluids 10:2026-2036

99. Yeung PK, Brasseur J, Wang Q. 1995. Dynamics of direct large-small scale couplings in coherently forced turbulence: concurrent physical- and Fourierspace views. J. Fluid Mech. 283:43-95

100. Yousef TA, Rincon F, Schekochihin AA. 2007. Exact scaling laws and the local structure of isotropic magnetohydrodynamic turbulence. J. Fluid Mech. $575: 111-120$ 
101. Zel'Dovich YB, Ruzmaikin AA, Molchanov SA, Sokoloff DD. 1984. Kinematic dynamo problem in a linear velocity field. J. Fluid Mech. 144:1-11

102. Zhou Y. 1993. Interacting scales and energy transfer in isotropic turbulence. Phys. Fluids A 5:2511-2524

103. Zhou Y, Matthaeus WH, Dmitruk P. 2004. Colloquium: magnetohydrodynamic turbulence and time scales in astrophysical and space plasmas. Rev. Mod. Phys. $76: 1015-1035$ 

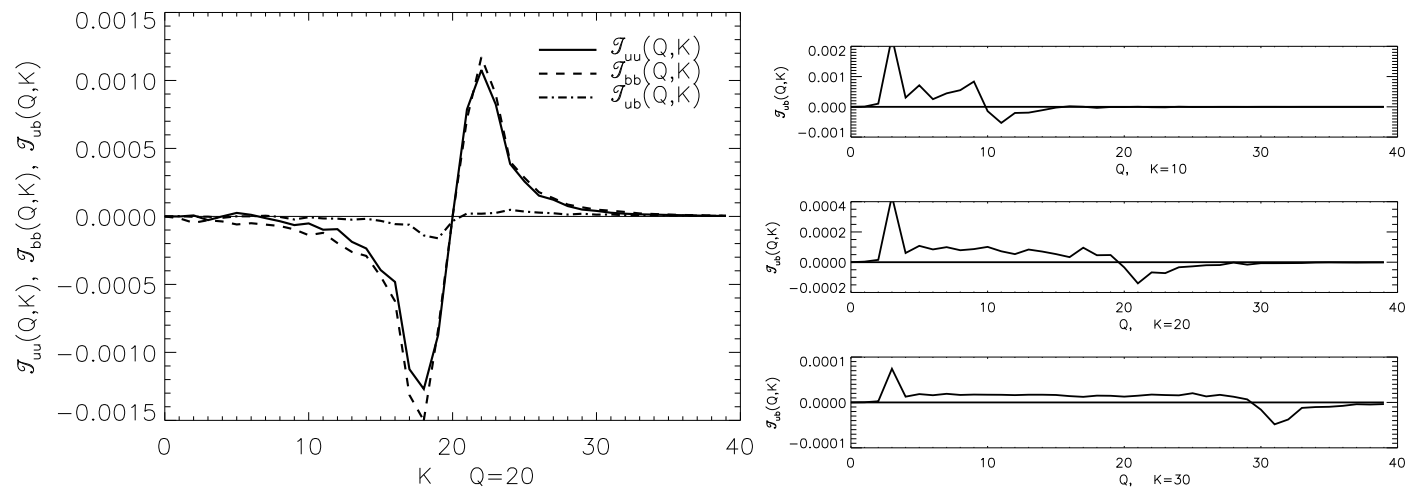

Figure 1: Left: transfer functions in mechanically forced MHD turbulence, for $Q=20$. The $T_{u u}$ and $T_{b b}$ functions are local, with a negative peak for $K<Q$ and a positive peak for $K>Q$, which indicate energy is removed by these transfer functions from smaller wavenumbers and given to slightly larger wavenumbers. The transfer between magnetic to kinetic energy is of smaller amplitude and also seems local. Right: The $T_{u b}$ transfer, for different values of $K$. This function is non-local, with a strong peak at the forcing scale and with a constant positive plateau that extends up to $K \approx Q$. Adapted from Alexakis et al. (2005b). 

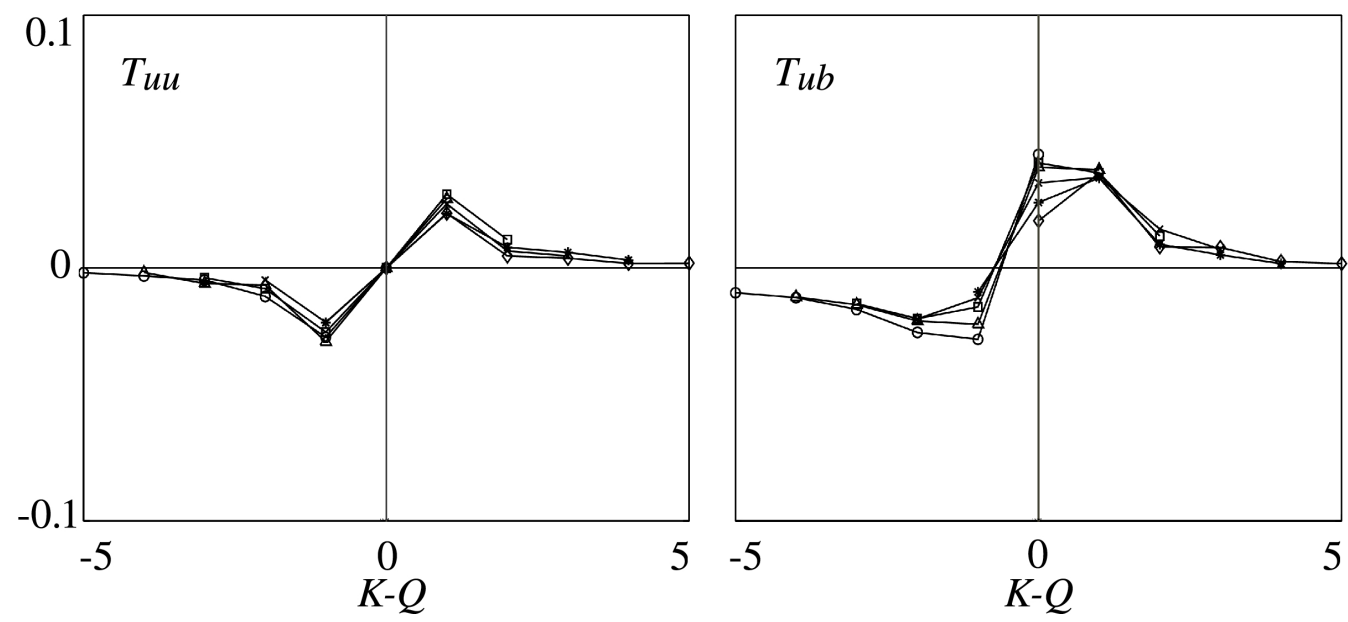

Figure 2: Left: $T_{u u}$ transfer function in freely decaying MHD turbulence, for different shells. The $T_{b b}$ transfer function is similar but has twice the amplitude. Right: $T_{b u}$ transfer function in the same simulation. Note the peak for $K-Q=0$, indicating most interchange of energy between the velocity and the magnetic field takes place between similar scales. The shells are logarithmically binned. Adapted from Debliquy et al. (2005). 

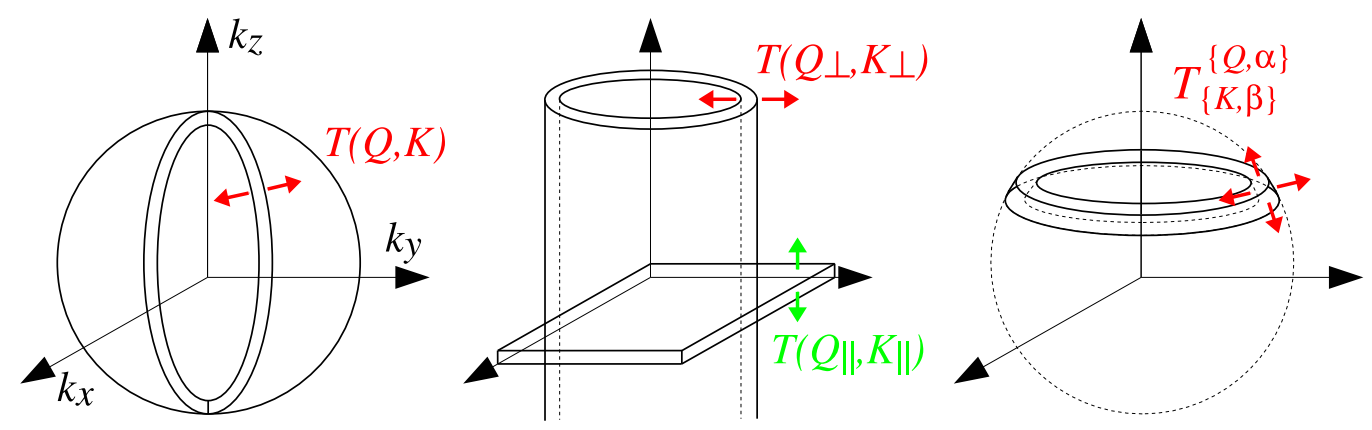

Figure 3: Isotropic (spherical) shells in the left, and anisotropic foldings of shells in Fourier space. The uniform magnetic field is assumed to be in the $z$ direction. Cylindrical and planar shells are shown in the middle, and ring shells are shown in the right. Transfer of energy across planes is denoted by $T\left(Q_{\|}, K_{\|}\right)$, and transfer across cylinders is denoted by $T\left(Q_{\perp}, K_{\perp}\right)$. For ring-to-ring transfers, the notation $T_{\{K, \beta\}}^{\{Q, \alpha\}}$ denotes transfer can be measured between $K$ and $Q$ spherical shells, as well as between two azimuthal angles $\alpha$ and $\beta$. 

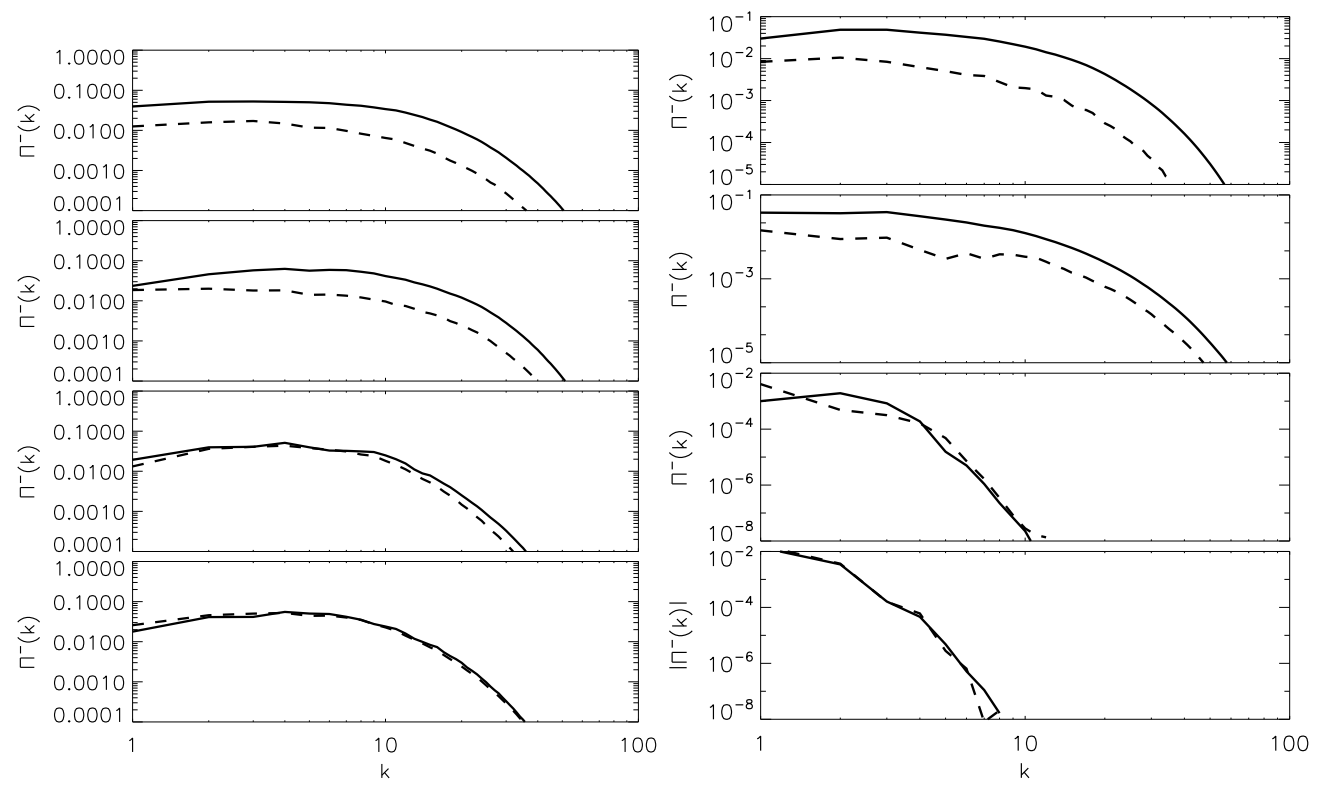

Figure 4: Left: total energy flux (solid) across cylinders and partial flux associated to interactions with modes with $k_{\|}=0$ (dashed), with four different values of the external magnetic field $B_{0}$ from 0 to 15 (from top to bottom). Right: same but with the total flux and partial flux associated to interactions with modes with $k_{\|}=1$ across planes. From Alexakis et al. (2007a). 

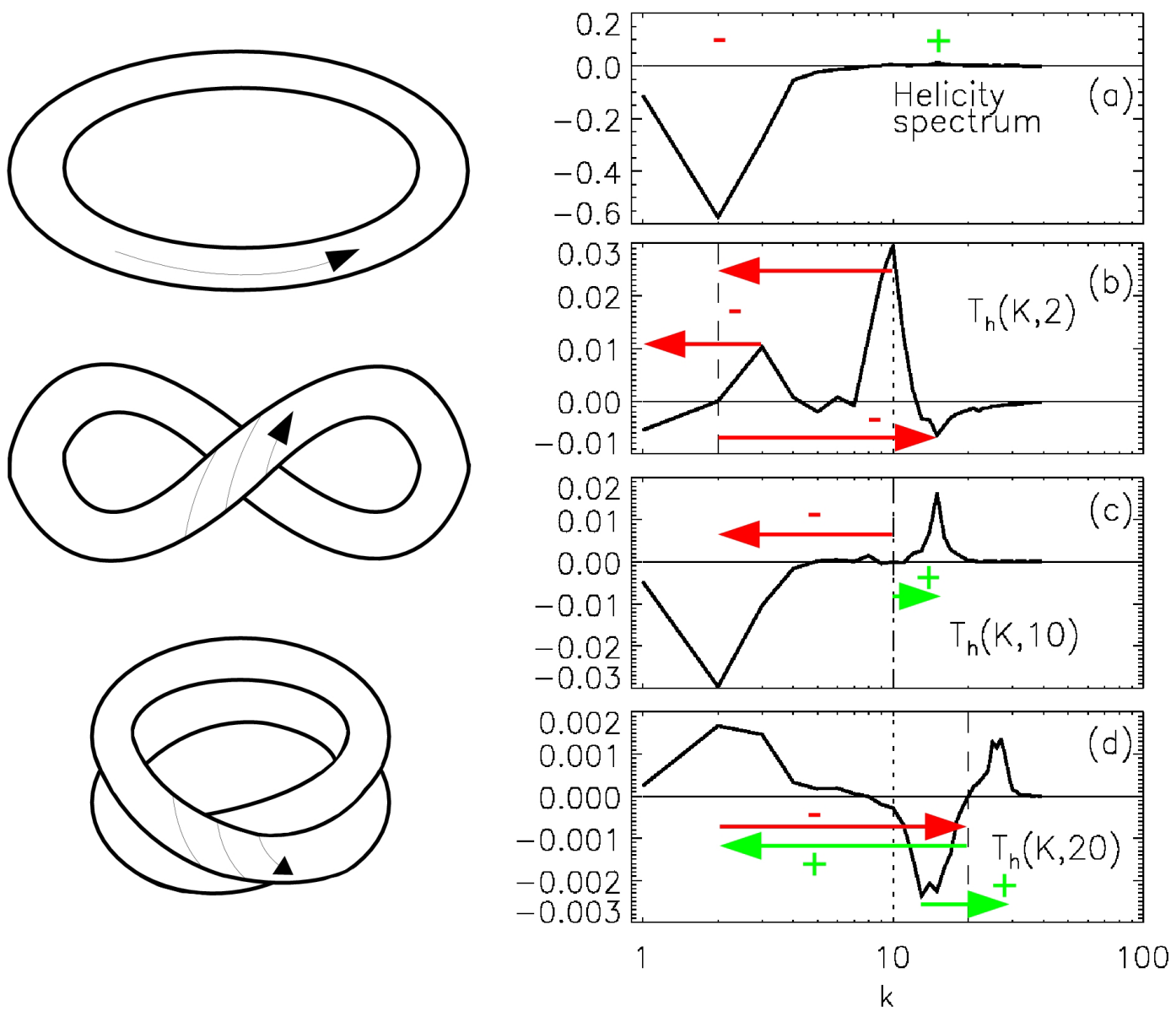

Figure 5: Left: the stretch, twist, and fold dynamo mechanism. Each time a closed magnetic flux tube is twisted, magnetic helicity of opposite sign is created at large and small scales. The folding creates regions where helical magnetic fields can reconnect. Right: (a) The helicity spectrum in a simulation with (positive) helical mechanical forcing at $k=10$. Magnetic helicity is negative at larger scales and positive at smaller scales. (b,c,d): The transfer of helicity for $Q=2,10$, and 20 . The red arrows indicate transfer of negative helicity and the green arrows transfer of positive helicity. At large scales (b), negative magnetic helicity inversely cascades locally between neighbor shells and non-locally from the forced shell and to the small scale shells. At the forced shell (c), the forcing injects opposite signs of helicity at large and small scales. At small scales (d), positive magnetic helicity has a local direct transfer of helicity, while the small scales also remove negative magnetic helicity from the large scales. Note direct 


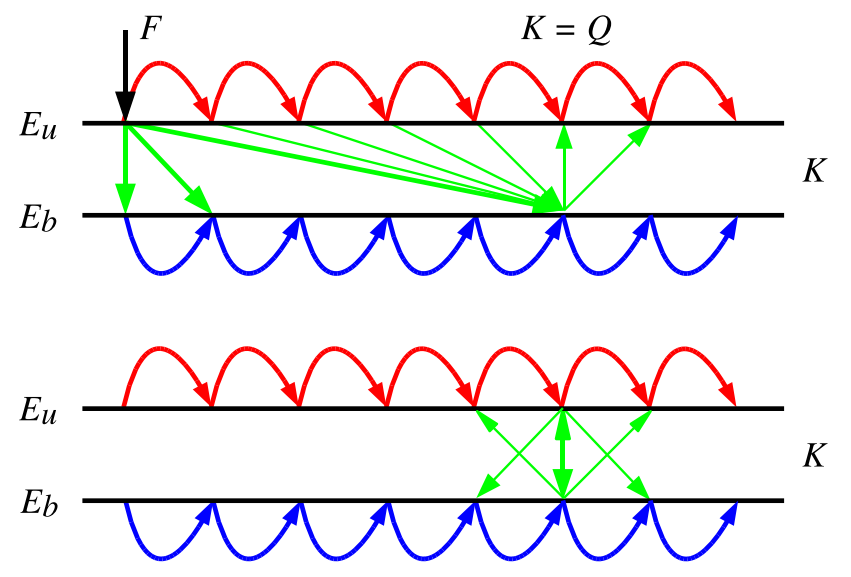

Figure 6: Sketch of the several shell-to-shell energy transfers identified in simulations of isotropic and homogeneous MHD turbulence. The $T_{u u}$ transfers are shown in red, $T_{b b}$ transfers are shown in blue, and $T_{u b}$ and $T_{b u}$ in green. The thickness of the arrows roughly indicates the strength of the transfers. Above: mechanically forced simulations. At the shell $K$, the magnetic field receives energy from the velocity field at all larger scales, and gives energy to the velocity field at slightly smaller scales. Below: Freely decaying turbulence. The $T_{u b}$ and $T_{b u}$ transfers only interchange energy between similar scales. In both cases, the $T_{u u}$ and $T_{b b}$ transfers are local and give the largest contribution to the flux. 


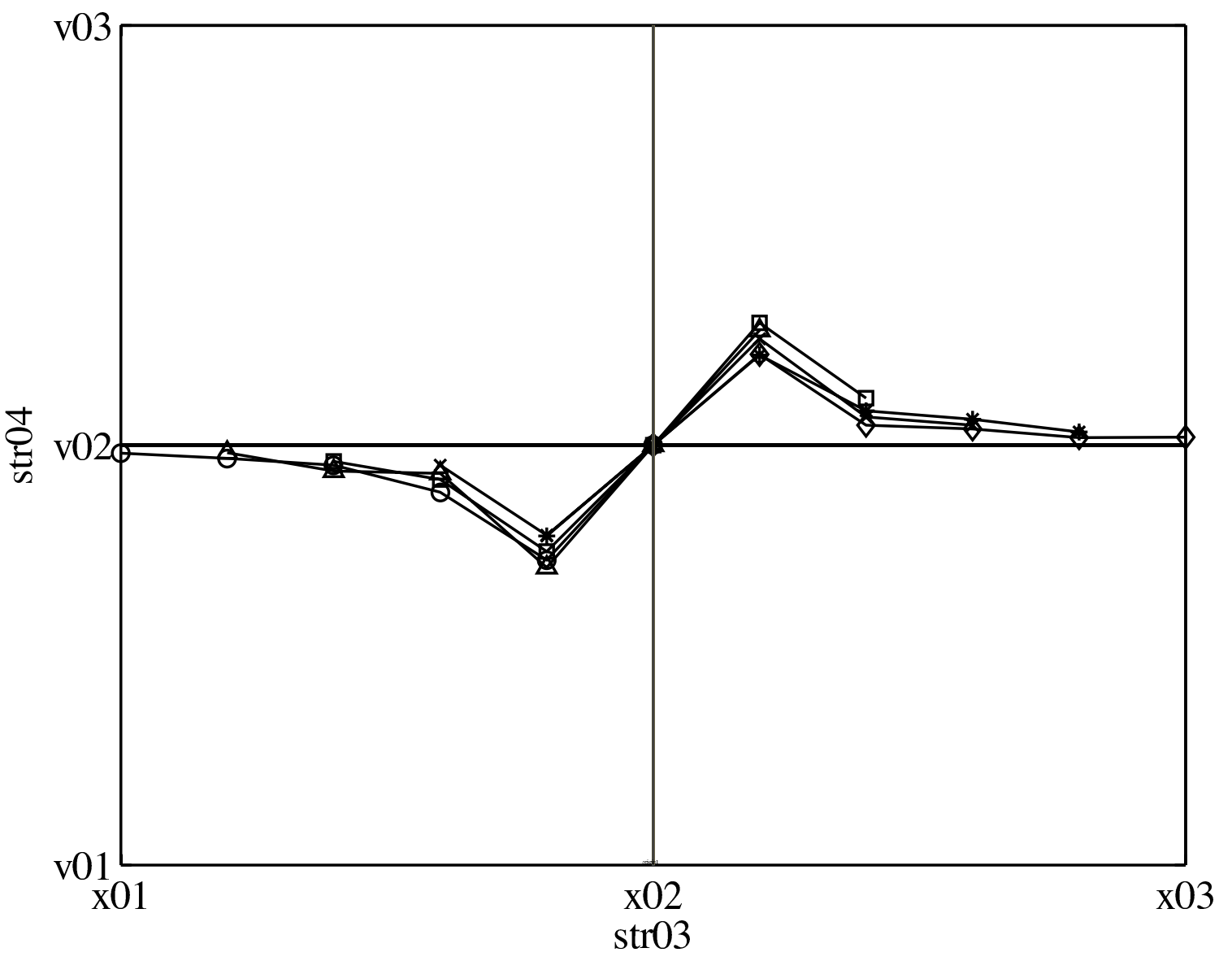


$\mu$ 